\title{
The optical afterglow of GRB 000911: Evidence for an associated supernova?^
}

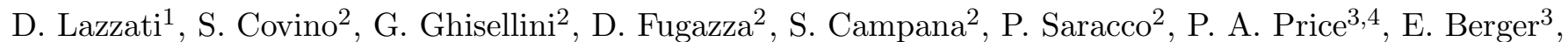 \\ S. Kulkarni ${ }^{3}$, E. Ramirez-Ruiz ${ }^{1}$, A. Cimatti ${ }^{5}$, M. Della Valle ${ }^{5}$, S. di Serego Alighieri ${ }^{5}$, A. Celotti ${ }^{6}$, \\ F. Haardt ${ }^{7}$, G. L. Israel ${ }^{8}$, and L. Stella ${ }^{8}$
}

1 Institute of Astronomy, University of Cambridge, Madingley Road, CB3 0HA Cambridge, UK

2 Osservatorio Astronomico di Brera, Via Bianchi 46, 23807 Merate (Lc), Italy

3 Palomar Observatory, 105-24, California Institute of Technology, Pasadena, CA 91125, USA

4 Research School of Astronomy \& Astrophysics, Mount Stromlo Observatory, Cotter Road, Weston, ACT 2611, Australia

5 Osservatorio Astrofisico di Arcetri, Largo E. Fermi 5, 50125 Firenze, Italy

6 SISSA/ISAS, via Beirut 4, 34014 Trieste, Italy

7 Università dell'Insubria, Via Lucini 3, 22100 Como, Italy

8 Osservatorio Astronomico di Roma, Via Frascati 33, 00040 Monteporzio Catone, Italy

Received 12 July 2001 / Accepted 12 September 2001

\begin{abstract}
We present photometric and spectroscopic observations of the late afterglow of GRB 000911, starting $\sim 1$ day after the burst event and lasting $\sim 8$ weeks. We detect a moderately significant re-brightening in the $R, I$ and $J$ lightcurves, associated with a sizable reddening of the spectrum. This can be explained through the presence of an underlying supernova, outshining the afterglow $\sim 30$ days after the burst event. Alternative explanations are discussed.
\end{abstract}

Key words. gamma rays: bursts - supernovae: general

\section{Introduction}

An anomalous re-brightening has been detected in the optical lightcurves of at least two gamma-ray bursts $\sim 30$ days after the burst event (GRB 980326: Bloom et al. 1999; GRB 970228: Reichart 1999; Galama et al. 2000). These have been tentatively interpreted as due to the simultaneous explosion of a supernova $(\mathrm{SN})$ with a lightcurve similar to that of SN 1998bw (Galama et al. 1998) which outshines the afterglow emission at the time of the SN peak. GRB 980326 showed a clear excess in $R$ band (aided by having a very faint host galaxy) and the spectrum changed from blue (early) to red close to the peak of rebrightening; however, neither the redshift of the afterglow nor the host is known to date (Bloom et al. 1999). GRB 970228 had the advantage of a known redshift

Send offprint requests to: D. Lazzati,

e-mail: lazzati@ast.cam.ac.uk

* Based on observations made with ESO Telescopes at the Paranal Observatories under programme IDs 65.H-0215 and 266.D-5620. Some of the data presented here were obtained at the W. M. Keck Observatory, which is operated as a scientific partnership among the California Institute of Technology, the University of California, and the National Aeronautics and Space Administration. The Observatory was made possible by the generous financial support of the W. M. Keck Foundation.
(Djorgovskij et al. 1999), a moderate amount of multiband data but lacked spectroscopic coverge. A broad band multifilter spectral energy distribution (SED) of GRB 970228 at day $\sim 30$ was extracted (Reichart 1999), but the $K$ band measurement, important to constrain the reddening, was questioned by Galama et al. (2000).

The confirmation of a GRB-SN connection is clearly of primary importance to solve the problem of the burst progenitor (Eichler et al. 1989; Woosley 1993; Paczyǹski 1998), but also bears important consequences on the $\gamma$-ray radiation mechanism (Lazzati et al. 2000) and the origin of emission and absorption features in the $\mathrm{X}$-ray spectra of the burst proper and its afterglow (Lazzati et al. 1999; Amati et al. 2000; Piro et al. 2000; Rees \& Meszaros 2000; Vietri et al. 2001; Lazzati et al. 2001).

Here we present the results of a simultaneous multifilter observational campaign designed at detecting and studying the spectrum of the re-brightening component in the burst of September 11th, 2000. We observed the optical transient (OT) in five filters at three epochs. A low resolution spectrum was taken $\sim 36$ days after the burst explosion.

GRB 000911 was detected by Ulysses, NEAR and Konus on September 11.30237 (Hurley 2000). It had a duration of $\sim 500 \mathrm{~s}$, a $25-100 \mathrm{keV}$ fluence of 
$\mathcal{F} \sim 5 \times 10^{-6} \mathrm{erg} \mathrm{cm}^{-2}$ and a peak flux over $0.5 \mathrm{~s}$ of $\sim 9 \times 10^{-7} \mathrm{erg} \mathrm{cm}^{-2} \mathrm{~s}^{-1}$. Its afterglow was detected at radio wavelengths $(8.46 \mathrm{GHz}$, Berger \& Frail 2000) and subsequently confirmed in the optical $R$ band (Berger et al. 2000) at a position of RA $02^{\mathrm{h}} 18^{\mathrm{m}} 34^{\mathrm{s}} .36$ and Dec $07^{\circ} 44^{\prime} 27^{\prime \prime} .7$ (Price 2000). The OT displayed a fairly rapid decay law $F_{\nu}(t) \propto t^{-1.4}$ (Price et al. 2000, see also Sect. 3.1) in the $R$ band. The redshift of the host galaxy was measured to be $z=1.06$ (Price et al. 2001).

\section{Observations}

We observed the OT associated with GRB 000911 with the ESO/VLT-Antu telescope (instruments, FORS1 and ISAAC), the Keck I telescope (instrument, LRIS) and the MSO 50-inch telescope; see Tables 1 and 2 for log of observations. The VLT observations were designed to optimize the chances of detecting a re-brightening due to a $\mathrm{SN}$ similar to that of SN 1998bw. Furthermore, we obtained low resolution spectra with the FORS1 instrument (grism 150I, blocking filter OG590) on day 36, around the time of the expected peak SN emission.

\subsection{Photometry}

All scientific frames were reduced in a standard way with the ESO-MIDAS package (98 NOV and 99 NOV versions). Photometric analysis was carried out with DAOPHOT II (Stetson 1987) as implemented in the ESO-MIDAS and IRAF (version 2.11.3) packages. We derived both aperture and point-spread function (PSF) fitting photometry. The model PSF was always based on a large number of stars. Seeing conditions were good in the first and third VLT runs, $(\sim 0.8$ arcsec $)$, and exceptional in the second one $(\sim 0.5$ arcsec $)$. For all three VLT runs suitable standard star fields were observed: SA98, Mark A and SA113 in the optical; FS28, S234 and P530 in the $J$ band. The observations were all performed under photometric conditions. A comparison of our photometric calibration and that by Henden (2000) shows that ours is slightly fainter ( $\sim 0.1 \mathrm{mag})$ for stars at the faint end of Henden's list.

The observation log and photometric measurements for the three multifilter VLT observations are reported in Table 1 . Table 2 shows the additional photometric measurements taken with the Keck and MSO50 telescopes, as well as two photometric points extracted from the VLT spectrum. All these measurements, together with two additional photometric points taken from the literature (see Table 3 ), are shown in Fig. 1. The OT appears point like in all filters at all times, with the exception of the $B$ and $V$ images at day 53 , in which the OT is not consistent with the PSF of bright stars. The faintness of the OT in $B$ and $V$ bands at day 53 , however, makes it impossible to measure a reliable extension and ellipticity of the source. The error bars of photometric points were derived following the standard procedure. All pertinent factors affecting photometric precision are taken into account: statistical errors on the measured counts converted to electron units, CCD read-out noise, calibration errors applying the usual error propagation formulas. On the other hand, the photometry of the OT was not affected by the galaxy background possibly present in our images. In fact, we did not detect significant evidence of deviation from the field star PSF for the OT (the only exception is for the last $B$ and $V$ images, see above). The underlying galaxy within the limit of our observational data seem to be consistent wih a point-like shape perfectly superposed (i.e. within a fraction of the $F W H M$ of our images) to the OT. After having removed all star-like objects in our images no diffused background emerged in the OT region. A systematic uncertainty of $0.05 \mathrm{mag}$ was added in quadrature to all data, in order to get rid of the differences possibly introduced by images taken with different telescopes.

\subsection{Spectroscopy}

The spectral frame reduction was again performed in a standard way with ESO-MIDAS tools (99 NOV version). The extraction of the scientific spectra was then obtained with IDL routines written by us. Observing conditions were photometric. However the closeness of the moon to the target $\left(\sim 30^{\circ}\right)$, the seeing variations (from $\sim 0.8$ arcsec to $\sim 1.8$ arcsec) and the OT faintness made the spectrum extraction critical. In particular it proved necessary to rebin our spectra to the much lower resolution of $110 \AA$ per pixel in order to increase the $\mathrm{S} / \mathrm{N}$. Two independent spectra were extracted from different frames, one hour exposure each, and found to be consistent with each other. Absolute calibrations were performed through the observation of a standard star (EG21) with the same instrumental set-up.

The resulting average spectrum is shown by the filled circles in Fig. 3. The spectrum, despite its very low resolution, was determined to be considerably redder than any afterglow spectrum observed so far. A power-law fit yielded $F(\nu) \propto \nu^{-5.3 \pm 0.8}\left(1 \sigma\right.$ error, $\chi^{2}=22$ for 21 degrees of freedom, hereafter d.o.f.).

\section{Data modelling}

We have modeled the data with a composite spectrum given by combining an external shock synchrotron component (Meszaros \& Rees 1997) plus a host galaxy. In addition, we examined the possible role of a supernova component. The details of the photometry and spectral fitting are discussed in the following subsections.

\subsection{Photometry}

The photometric data were dereddened for Galactic extinction according to the maps of Dickey \& Lockman (1990) $\left(E_{B-V}=0.112\right)$ and following extinction curves from Cardelli et al. (1989), and converted to flux densities. Extinction in the host galaxy was not modeled since no additional extinction was required by the data. 


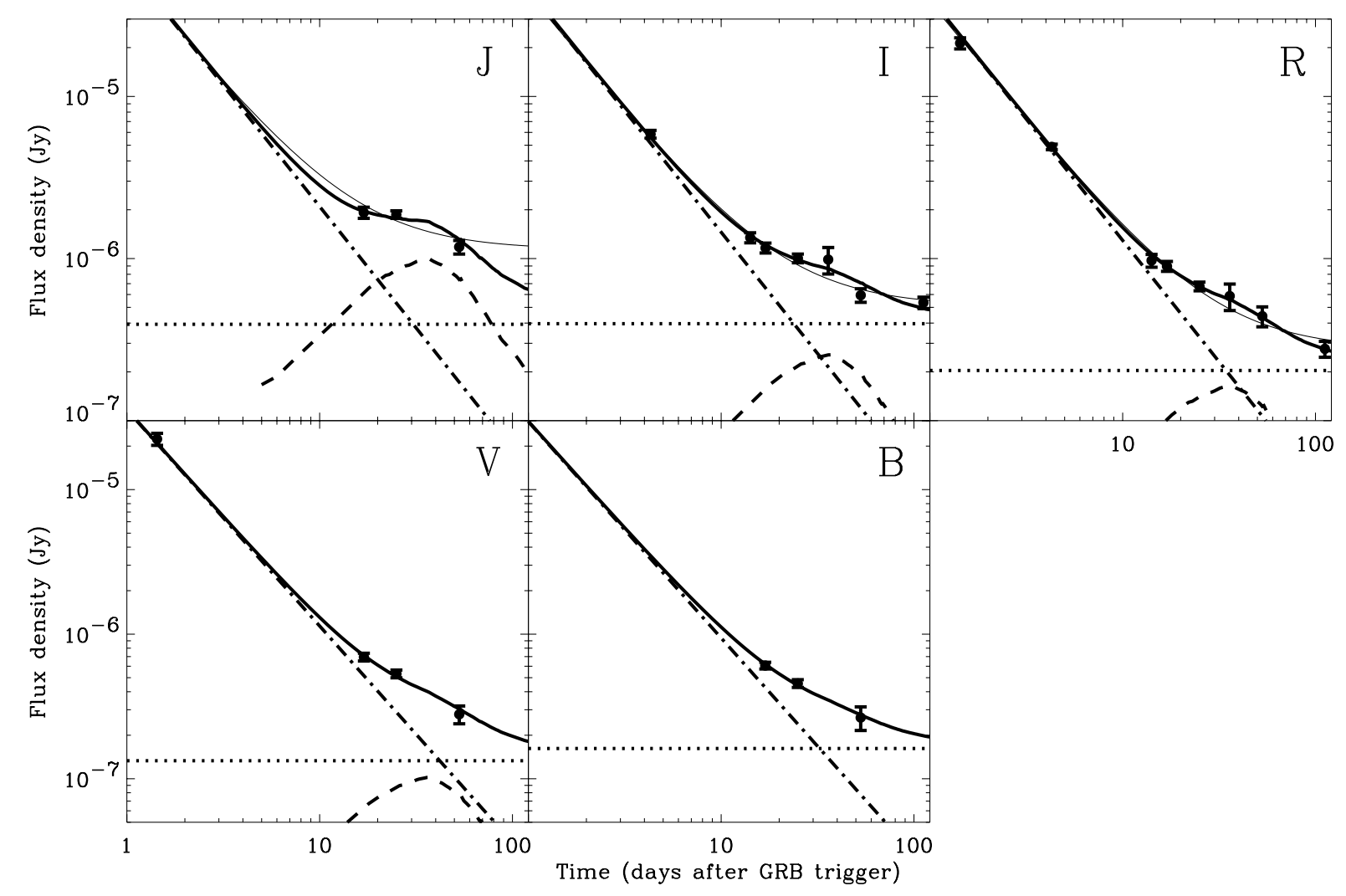

Fig. 1. Lightcurves of the afterglow of GRB 000911. From top left to bottom right, the $J, I, R, V$ and $B$ lightcurves are plotted. The thick solid curves show the best fits obtained with our three component model. The dashed, dotted and dot-dashed lines show the SN, galaxy and ES components, respectively. The thin solid lines in the $J, I$ and $R$ panels indicate the best fit for a model comprising only the galaxy and ES (without SN). The thin line is indistinguishable from the thick solid line for the $V$ and $B$ filters.

The lightcurve modelling was obtained as the sum of the above three contributions. First we considered an external shock afterglow component (hereafter ES) of the form:

$F(\nu, t)=A_{\mathrm{ES}} \nu^{-\alpha} t^{-2 \alpha}$.

This equation holds for a jet geometry after the break time (Sari et al. 1999). Such a configuration is obtained by a broadband fitting of the GRB 000911 afterglow (Price et al. 2001). The constant flux from a host galaxy was added in the five bands as a free parameter $\left(G_{B}, G_{V}, G_{R}\right.$, $G_{I}$ and $G_{J}$ ).

First, an ES plus galaxy model was fitted to the data. The best fit gave a decay slope $\alpha=0.724 \pm 0.006$ (temporal slope $\delta=1.45 \pm 0.012$ ) with $\chi^{2}=24.4$ for 18 d.o.f. This is an acceptable fit, with chance probability of $P \sim 10 \%$ to obtain a higher $\chi^{2}$ value. However, the fit can be improved by adding the SN component at the redshift $z=1.06$ of the host galaxy (see Sect. 1). The lightcurve of the supernova component was obtained by spline interpolation of the data of SN 1998bw ( $z=0.0085$; Galama et al. 1998). Cosmological parameters $H_{0}=65 \mathrm{~km} \mathrm{~s}^{-1} \mathrm{Mpc}^{-1}$ and $q_{0}=0.5$ were adopted to compute the flux as a function of redshift, and the time profile was stretched by a factor $1+z$. Following Bloom et al. (1999), the spectrum of the supernova was analytically extended in the rest frame ultraviolet assuming a power-law $F(\nu) \propto \nu^{-2.8}$ for $\lambda<3600 \AA$. SN1998bw was adopted as a template since it is the SN with the most widely accepted association with a GRB (GRB 980425) due to the coincident position (Galama et al. 1998) and its peculiar relativistic expansion (Kulkarni et al. 1998). In addition, it has a very well sampled UBVRI lightcurve (Galama et al. 1998).

The addition of the supernova component changes slightly the spectral slope of the ES component $(\alpha=$ $0.748 \pm 0.006)$ with $\chi^{2}=13.9(P \sim 67 \%)$. In principle, the addition of this component does not introduce any new free parameter since the redshift is known and the SN luminosity fixed to the value of SN 1998bw. However, the lightcurves of different $\mathrm{SNe}$, even within the same class, are different, with different peak luminosity and peak time. We hence let the normalization of the SN component free to vary, with the redshift, the color and the time profile held fixed. We obtain a normalization factor $A=0.9 \pm 0.3$, with $\chi^{2}=13.8$ for 17 d.o.f. An F test applied gives a statistical confidence of $99.8 \%(2.9 \sigma)$ for the fit improvement. Alternatively, we allowed the redshift to vary. Interestingly, we obtain $z_{\mathrm{SN}}=1.1$, in good agreement with the $z=1.06$ measured spectroscopically by Price et al. (2001) for the host galaxy of GRB 000911.

The re-brightening component in the lightcurve is hence remarkably similar in luminosity, shape and color 
Table 1. Log of the VLT photometric observations of the afterglow of GRB 000911.

\begin{tabular}{|c|c|c|c|l|c|}
\hline Date (UT) & $\Delta t(\mathrm{~d})$ & Filter & Exposure time $(\mathrm{s})$ & Magnitude & Seeing \\
\hline \hline 28 Sep. 2000 & 16.94 & $B$ & 2600 & $25.10 \pm 0.05$ & $0.8^{\prime \prime}$ \\
06 Oct. 2000 & 24.98 & $B$ & 2600 & $25.41 \pm 0.06$ & $0.6^{\prime \prime}$ \\
03 Nov. 2000 & 52.95 & $B$ & 2600 & $26.0 \pm 0.2$ & $1.0^{\prime \prime}$ \\
28 Sep. 2000 & 16.96 & $V$ & 1500 & $24.67 \pm 0.06$ & $0.8^{\prime \prime}$ \\
06 Oct. 2000 & 25.02 & $V$ & 1500 & $24.96 \pm 0.06$ & $0.5^{\prime \prime}$ \\
03 Nov. 2000 & 52.99 & $V$ & 1500 & $25.66 \pm 0.15$ & $0.7^{\prime \prime}$ \\
28 Sep. 2000 & 16.98 & $R$ & 900 & $24.14 \pm 0.07$ & $0.8^{\prime \prime}$ \\
06 Oct. 2000 & 25.03 & $R$ & 900 & $24.45 \pm 0.06$ & $0.5^{\prime \prime}$ \\
03 Nov. 2000 & 53.00 & $R$ & 900 & $24.91 \pm 0.15$ & $0.7^{\prime \prime}$ \\
28 Sep. 2000 & 16.99 & $I$ & 1500 & $23.57 \pm 0.07$ & $0.8^{\prime \prime}$ \\
06 Oct. 2000 & 25.00 & $I$ & 1500 & $23.73 \pm 0.06$ & $0.5^{\prime \prime}$ \\
03 Nov. 2000 & 52.97 & $I$ & 1500 & $24.3 \pm 0.1$ & $0.7^{\prime \prime}$ \\
28 Sep. 2000 & 16.89 & $J$ & 2408 & $22.38 \pm 0.08$ & $0.7^{\prime \prime}$ \\
06 Oct. 2000 & 24.93 & $J$ & 2700 & $22.41 \pm 0.05$ & $0.5^{\prime \prime}$ \\
05 Nov. 2000 & 54.90 & $J$ & 2700 & $22.9 \pm 0.1$ & $0.5^{\prime \prime}$ \\
\hline
\end{tabular}

Table 2. Additional photometric points from Keck, MSO 50 inch telescope and VLT spectroscopy.

\begin{tabular}{|c|c|l|c|l|c|}
\hline$\Delta t(\mathrm{~d})$ & Filter & Telescope & Exposure time $(\mathrm{s})$ & Magnitude & Seeing \\
\hline \hline 1.435 & $R$ & MSO50 & 1200 & $20.70 \pm 0.08$ & $2.1^{\prime \prime}$ \\
1.435 & $V$ & MSO50 & 1200 & $20.90 \pm 0.1$ & $2.1^{\prime \prime}$ \\
4.30 & $R$ & Keck & 600 & $22.30 \pm 0.06$ & $0.6^{\prime \prime}$ \\
4.31 & $I$ & Keck & 620 & $21.82 \pm 0.06$ & $0.7^{\prime \prime}$ \\
14.12 & $R$ & Keck & 1830 & $24.05 \pm 0.094$ & $1.1^{\prime \prime}$ \\
14.15 & $I$ & Keck & 1080 & $23.41 \pm 0.07$ & $0.9^{\prime \prime}$ \\
35.92 & $R$ & VLT Spectrum & 6800 & $24.6 \pm 0.2$ & $0.9^{\prime \prime}$ \\
35.92 & $I$ & VLT Spectrum & 6800 & $23.75 \pm 0.2$ & $0.9^{\prime \prime}$ \\
\hline
\end{tabular}

Table 3. Photometric points from literature.

\begin{tabular}{|c|c|c|c|l|c|}
\hline$\Delta t(\mathrm{~d})$ & Filter & Telescope & Magnitude & Reference & Seeing \\
\hline \hline 111.9 & $R$ & Keck & $25.4 \pm 0.1$ & Price et al. (2001) & $0.7^{\prime \prime}$ \\
112.0 & $I$ & Keck & $24.4 \pm 0.1$ & Price et al. (2001) & $0.65^{\prime \prime}$ \\
\hline
\end{tabular}

to the lightcurve of SN 1998bw. As a final test, we allowed for a temporal shift $\Delta t$ between the SN and GRB explosions. Keeping all the other SN parameter fixed to the values of SN1998bw and the redshift $z=1.06$, we obtain $\Delta t=0_{-7}^{+1.5}$ days $(1 \sigma)$, showing that the SN explosion may anticipate the GRB but only by $\sim 1$ week (see, e.g., the Supranova model by Vietri \& Stella 1998).

The best fit three component model (ES plus galaxy plus SN) is shown by the thick solid line in Fig. 1. The figure also shows the three individual components (ES, galaxy and $\mathrm{SN})$.

In Fig. 2 we report the evolution of the color index $R-I$ as a function of time. The color significantly evolves from blue to red, as time goes on. A formal best fit with a constant color yields $\chi^{2}=23$ for 5 d.o.f. The reddening behavior can be due either to the presence of an underlying supernova or to a red host galaxy $(R-I \sim 1)$ which certainly dominates the color evolution at late time $(t \sim$ $100 \mathrm{~d}$ ). Again, the quality of the data, especially at late times, does not allow us to unambiguously select a model.

\subsection{Spectroscopy}

According to the fits described above, none of the three components clearly dominated the emission at the time of the spectroscopic observation ( $\sim 36$ days; see Fig. 1). Moreover, the wavelength range over which the spectrum has a sufficient signal to noise to be safely extracted covers only a limited spectral range $(6000 \AA<\lambda<8500 \AA)$. 


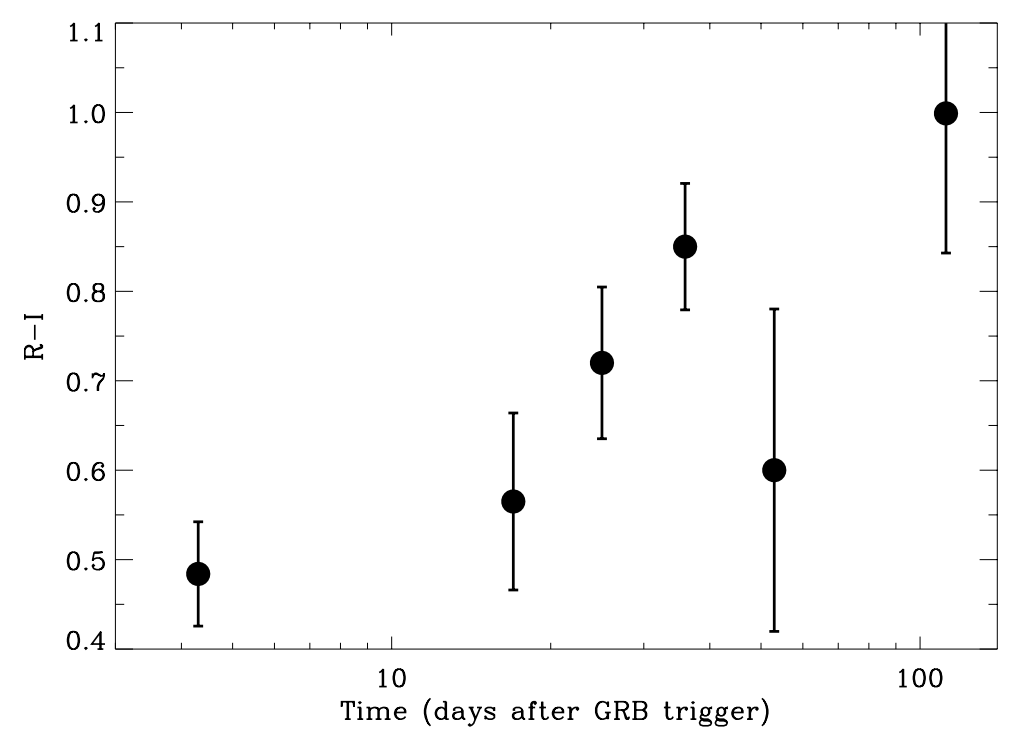

Fig. 2. Evolution of the $R-I$ color index with the time elapsed after the GRB event. The color shows a significant evolution from the value $R-I \sim 0.55$ in the first 20 days to the final value of $R-I \sim 0.85$ afterwards.

For this reason, the spectrum by itself can be modeled in different ways, all consistent with the data.

To better constrain the models, we added photometric information to the spectrum. In the upper panel of Fig. 3, the spectrum (filled dots) is plotted together with the $J$ band measurement at day 25 (diamond). Note that the change in the $J$ magnitude from day 25 to day 36 is expected to be small (see the first panel of Fig. 1). In the lower panel, the best fit galaxy components obtained with the lightcurve fitting procedure (from left to right $G_{B}, G_{V}$, $G_{R}, G_{I}$ and $\left.G_{J}\right)$ are shown for the five photometric filters used (triangles). We modeled the ensemble of these data with a galaxy spectrum (templates from Calzetti et al. 1994) plus a power-law ES spectrum (parameters fixed to the best-fit values from the lightcurve) and a type Ic supernova spectrum a few days after the peak (SN 1987M, Filippenko et al. 1990) ${ }^{1}$.

The two models were fitted as follows: the total template spectrum was fitted to the spectral data together with the $J$ band measurement; galaxy magnitudes derived from the template galaxy were fitted to the data in the lower panel. For the galaxy plus ES model, a formal best fit was obtained with a dust-enshrouded starburst galaxy template. The fit gave $\chi^{2}=58$ for 27 d.o.f. $(P \sim 0.05 \%)$. A better fit $\left(\chi^{2}=37\right.$ for 27 d.o.f., $\left.P \sim 9 \%\right)$ was obtained by adding a SN component, with a moderately dust-enshrouded starburst galaxy $\left(0.11<E_{B-V}<0.21\right)$ as a template. The fit is shown in Fig. 3 overlaid on the data.

In order to obtain a single statistical indicator combining the photometric and spectral information, we finally fitted simultaneously all the available data with the appropriate galaxy template. This yielded $\chi^{2}=44$ (39 d.o.f.)

\footnotetext{
${ }^{1}$ A spectrum of SN 1998bw would be more suited for this comparison, however no spectra of SN 1998bw at peak extend to wavelengths $\lambda<3500 \AA$, a range that is necessary for a proper comparison with the data of a SN at $z=1.06$.
}

and $\chi^{2}=69$ (40 d.o.f.) for the models with and without SN component, respectively. The $\chi^{2}$ decrease has a statistical significance of $4 \sigma$, according to the $F$-test.

\section{Alternatives}

We detected a possible re-brightening and reddening of the OT associated with GRB $000911 \sim 30$ days after the GRB event. We showed that this is consistent with the presence of an underlying SN component. In the following we discuss two possible alternatives.

\subsection{Dust echoes}

It has been suggested that the re-brightening detected in the late optical lightcurves of several GRBs may be explained by dust scattering or reprocessing of the burst and/or afterglow photons (Waxman \& Draine 2000; Esin \& Blandford 2000). The spectrum and lightcurve of these components have been modeled in detail by Reichart (2001). He concludes that the re-brightening in the lightcurve of GRB 970228 cannot be explained with dust scattering and reprocessing, because it predicts a spectral shape different from the observed one.

The same conclusion can be drawn in the case of GRB 000911. In fact, the re-brightening due to dust reprocessing has an exponential cut-off at wavelengths $\lambda<2.2(1+z) \mu \mathrm{m}$ (Reichart 2001), which lies in the Johnson $L$ filter for a $z=1.06$ burst. We detect a rebrightening in the $R, I$ and $J$ filter, and hence this interpretation can be ruled out. Scattering from dust, on the other hand, does not change the spectral index of the afterglow by more than $\sim 0.5$ for intermediate-low dust opacities (Reichart 2001). The spectrum we measure at day 36 implies a much larger reddening (see Sect. 2.2) and a dust echo for a low-intermediate dust opacity $\tau_{\text {dust }} \lesssim 12$ cannot be fitted to the data even considering the contribution 


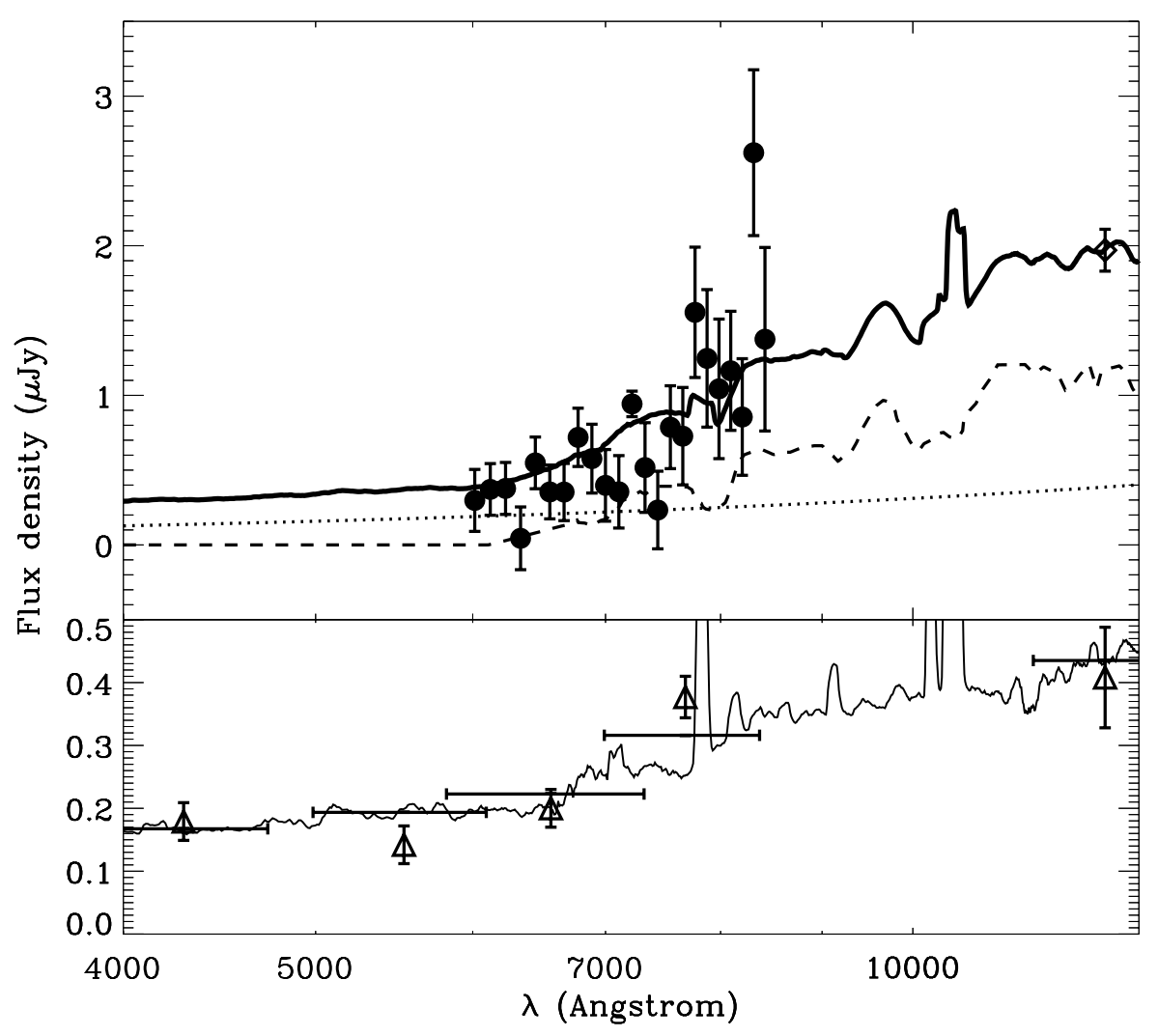

Fig. 3. Spectrum of the OT of GRB 000911 observed 36 days after the burst explosion (upper panel, filled dots). The spectrum is modelled with a SN type Ic spectrum plus a background starburst galaxy and an ES component (see text). The solid line shows the total spectrum smoothed with a $110 \AA$ boxcar filter, while the dashed line represents the supernova component (SN 1987M, see text). The lower panel shows the template spectrum of the best fit galaxy model (thin solid line). Triangles are the galaxy photometric measurements as derived from the multiband fitting (see Sect. 3.1 and the dotted horizontal lines in Fig. 1). The vertical position of the horizontal bars indicate the BVRIJ filter fluxes derived from the galaxy template; their width is equal to the full width at half maximum of the filters.

from a red host galaxy (the fit yields a $\chi^{2}$ comparable to that obtained for the ES plus galaxy model). For a $\tau_{\text {dust }}>12$ dust cloud, the re-brightening would be so small that it would remain undetected. The above conclusion can be clearly further strengthened through the measurement of the $J$ magnitude of the host galaxy, which would allow us to better constrain the temporal and spectral shape of the re-brightening component.

\subsection{Wind-fireball interaction}

In the collapsar model for GRBs (MacFadyen \& Woosley 1999), or in any other model involving a massive star, the key to obtain relativistic motions is the escape of an energy-loaded fireball from the stellar environment. A focused low-entropy jet that has broken free of its stellar cocoon is likely to arise from a Wolf-Rayet (WR) progenitor. WR stars are characterized by strong stellar winds gradually shedding most of the star envelope. The deceleration of this pre-collapse wind by the pressure of the surrounding medium causes the ejected mass to accumulate at a radius $\sim 10^{17} \dot{M}_{-4}^{1 / 2} n_{0,1}^{-1 / 2} v_{3}^{-1 / 2} \mathrm{~cm}$ (where $\dot{M}$ is the mass loss rate in units of solar masses per year, $n_{0}$ is the density of the surrounding medium in units of $\mathrm{cm}^{-3}$, $v$ the wind velocity in units of $\mathrm{km} \mathrm{s}^{-1}$ and we adopt the convention $\left.Q=10^{x} Q_{x}\right)$. The impact between the forward shock and these high-density regions should be observed as a re-brightening of the afterglow, typically with a redder spectrum. Using detailed stellar tracks for the evolution of massive stars, as described in Ramirez-Ruiz et al. (2001), we found that the re-brightening of GRB 000911 at $z=1.06$ can be explained by the interaction of a relativistic blast wave expanding into the ambient medium expected at the end of the life of a $40 M_{\odot}\left(7 M_{\odot}\right.$ core) WR star evolving with solar metallicity. The shock front expands within a $n(r)=1.5 \times 10^{35} r^{-2} \mathrm{~cm}^{-1}$ stellar wind until it reaches the density enhancement (see Fig. 3 of Ramirez-Ruiz et al. 2001). If we consider the forward shock emission alone (shock wave into the accumulated wind), it is not possible to have a spectral slope steeper than $\alpha \sim 3.5$ (Ramirez-Ruiz et al. 2001). Including the emission of the reverse shock, spectral slopes as steep as $\alpha \sim 4$ can be obtained, depending on the radiative efficiency of the reverse shock. The spectroscopic observation can then be reproduced if some of the reddening is attributed to the host galaxy emission.

We have fitted this model to the photometric data only obtaining a $\chi^{2}$ intermediate between the fit with a 
simple ES plus galaxy model and the fit with the SN additional component. This model also requires the addition of 4 free parameters (the Lorentz factor of the fireball, the mass of the progenitor star, its metallicity and the density of the surrounding medium) and the need for an enhanced reverse shock emission to reproduced the observed spectrum. Since the addition of more degrees of freedom does not improve the statistics of the $\chi^{2}$, we consider the SN a better explanation for the re-brightening in the GRB 000911 lightcurve.

\section{Conclusions}

We presented late time multifilter observations of the optical transient associated to GRB 000911. This set of observations was designed to detect and analyze the re-brightening associated with (some) GRB afterglows approximately one month after their explosion (Bloom et al. 1999; Reichart 1999; Galama et al. 2000). In addition to photometric data, a low resolution spectrum was taken $\sim 36$ days after the burst explosion.

The lightcurve and spectrum were fitted with an external shock plus galaxy model, with the possible addition of a supernova, similar to SN 1998bw. The addition of the SN component gives a better fit, with a statistical significance of $4 \sigma$. Even though the observations were designed to detect unambiguously the additional supernova component, the signal to noise ratio that could be achieved was limited, due to the combination of two effects. Firstly the burst was at a redshift larger than in the two previous cases (GRB 970228: $z=0.695$; GRB 980326: $z \sim 0.9$ ); secondly, the host galaxy associated with GRB 000911 had a brightness comparable to that of the SN component. For this reason, a fourth multifilter observation at a much longer time (>100 days) is mandatory in order to disentangle the two components. We were able to find $R$ and $I$ magnitudes at this late times in the literature (Price et al. 2001), but the most important measurement in the $J$ band (see the difference between the thick and thin solid lines in the $J$ panel of Fig. 1) was not available.

With the present data, a word of caution should be spent, since is not possible to unambiguously assess the presence of the SN. However, if future observations will allow us to better constraint the magnitude of the host galaxy and will confirm the presence of the rebrightening, we will be able to disentangle the SN component and to provide simultaneous multiband SEDs at the time of our 3 VLT observations. Such time resolved broad band SEDs will allow to better understand the spectral evolution of the bump in the lightcurve and hence to understand its physical origin.

Acknowledgements. We thank the ESO-ANTU service team that performed the observations. We thank the ESO Director for the allocation of discretionary time for the spectral observations performed on day 36 . This program was partially supported through ASI grants. SRK thanks NSF and NASA for supporting a program of GRB investigations at Caltech.

\section{References}

Amati, L., Frontera, F., Vietri, M., et al. 2000, Science, 290, 953

Berger, E., \& Frail, D. A. 2000, GCN \#794

Berger, E., Price, P. A., Frail, D. A., et al. 2000, GCN \#795

Bloom, J. S., Kulkarni, S. R., Djorgovski, S. G., et al. 1999, Nature, 401, 453

Calzetti, D., Kinney, A. L., \& Storchi-Bergmann, T. 1994, ApJ, 429, 582

Cardelli, J. A., Clayton, G. C., \& Mathis, J. S. 1989, ApJ, 345, 245

Dickey, J. M., \& Lockman, F. J. 1990, ARA\&A, 28, 215

Djorgovski, S. G., Kulkarni, S. R., Bloom, J. S., \& Frail, D. A. 1999, GCN \#289

Eichler, D., Livio, M., Piran, T., \& Schramm, D. N. 1989, Nature, 340, 126

Esin, A. A., \& Blandford, R. 2000, ApJ, 534, L151

Filippenko, A. V., Porter, A. C., \& Sargent, W. L. W. 1990, AJ, 100, 1575

Galama, T. J., Vreeswijk, P. M., Van Paradijs, J., et al. 1998, Nature, 395, 670

Galama, T. J., Tarvir, N., Vreeswijk, P. M., et al. 2000, ApJ, 536,185

Henden, A. A. 2000, GCN \#800

Hurley, K. 2000, GCN \#791

Kulkarni, S. R., Frail, D. A., Wieringa, M. H., et al. 1998, Nature, 395, 663

Lazzati, D., Campana, S., \& Ghisellini, G. 1999, MNRAS, 304, L31

Lazzati, D., Ghisellini, G., Celotti, A., \& Rees, M. J. 2000, ApJ, 529, L17

Lazzati, D., Ghisellini, G., Amati, L., et al. 2001, ApJ, 556, 471

MacFadyen, A. I., \& Woosley, S. E. 1999, ApJ, 524, 262

Meszaros, P., \& Rees, M. J. 1997, ApJ, 476, 232

Paczyński, B. 1998, ApJ, 494, L45

Piro, L., Garmire, G., Garcia, M., et al. 2000, Science, 290, 955

Price, P. A. 2000, GCN \#799

Price, P. A., Galama, T. J., Goodrich, R. W., \& Diercks, A. 2000, GCN \#796

Ramirez-Ruiz, E., Dray, L. M., Madau, P., \& Tout, C. A., MNRAS, in press [astro-ph/0012396]

Rees, M. J., \& Meszaros, P. 2000, ApJ, 545, L73

Reichart, D. E. 1999, ApJ, 521, L111

Reichart, D. E. 2001, ApJ, 554, 643

Sari, R., Piran, T., \& Halpern, J. P. 1999, ApJ, 519, L17

Stetson, P. B. 1987, PASP, 99, 191

Vietri, M., \& Stella, L. 1998, ApJ, 507, L45

Vietri, M., Ghisellini, L., Lazzati, D., Fiore, F., \& Stella, L. 2001, ApJ, 550, L43

Waxman, E., \& Draine, B. T. 2000, ApJ, 537, 796

Woosley, S. E. 1993, ApJ, 405, 273 